and the intracellular domains where kept murine to enable salt bridges interaction, interaction with the CD3 zeta and the signaling into mouse cells.

Results T cells from CD3 epsilon epitope humanized mice are found in comparable frequency in spleen, blood and bone marrow from WT mice. B cells, monocytes, dendritic cells and NK frequencies are also similar to the frequencies of these cell types in WT mice, suggesting that the humanization of the epitope of CD3 epsilon did not alter the immune cells distribution in these mice. Activation of $\mathrm{T}$ cells with antibodies targeting human CD3 (clone SP34) induced CD4 and CD8 T cell proliferation, as well as production of IL-2 and IFNgamma. The CD3 functionality was demonstrated in vitro by the ability of $\mathrm{B}$ cells to produce $\operatorname{IgM}$ upon activation of $\mathrm{T}$ cells, suggesting a proper cooperation between $\mathrm{T}$ and $\mathrm{B}$ cells. Additionally, a first class of T-cell engagers targeting both human CD3 and a tumoral antigen, induced tumor cell lysis of MC38-Ag in a concentration-dependent manner. A second class of $\mathrm{T}$ cell engagers, also targeting $\mathrm{CD} 3$ and a tumoral antigen, showed an anti-tumor effect in vivo, and this effect was also shown to be dose-dependent.

Conclusions These data suggest that the CD3 epsilon N-terminal epitope humanized mouse model enables the assessment of efficacy and mechanism of action of T-cell engagers.This model is currently being intercrossed with immunostimulatory humanized mouse models to provide new opportunities for assessment of bi-specific antibodies targeting the CD3 and immunostimulatory molecules. This model is the first generation of a broader program aiming at developping a Pan CD3 humanized model, where the gamma, delta and epsilon chains of the CD3 complex will be humanized. The Pan CD3 humanized mice are currently being investigated for immune responses and would provide a broader tool for assessment of T-cell engagers.

http://dx.doi.org/10.1136/jitc-2020-SITC2020.0014

\section{A NOVEL CD28 HUMANIZED MOUSE MODEL FOR EFFICACY ASSESSMENT OF CD28-TARGETING THERAPIES}

Fabiane Sônego*, Gaelle Martin, Chloé Beuraud, Audrey Beringer, Yacine Cherifi, Alexandre Fraichard, Patricia Isnard-Petit, Kader Thiam. GeOway, Lyon, France

Background Immuno-intervention through targeting of activating and inhibitory immune checkpoints (ICP), has shown promising results in the clinic over the last years. To facilitate these researches, mouse models expressing humanized ICP instead of their mouse counterparts were developed. Herein, we describe a novel CD28 humanized mouse model (hCD28 model), designed to test compounds targeting human CD28 (hCD28).

Methods Human and mouse CD28 (mCD28) have different signalling responses, with hCD28 being known for inducing higher levels of pro-inflammatory cytokines upon stimulation with ligands/superagonists. This can be explained by the expression of CD28i, a hCD28 amplifier isoform which is not found in mouse. Additionally, evidences suggested that the different signalling between human and $\mathrm{mCD} 28$ relies on one amino acid change in the intracellular domain (ICD). ${ }^{1}$ Because the hCD28 model was developed to assess hCD28-targeting therapeutics, we decided to keep the expression of both canonical and CD28i isoforms to avoid undermining the biological effects of the testing antibodies. Although keeping the human ICD could favour the evaluation of cytokine production and therefore the safety of the test therapeutics, we decided to keep the mouse ICD to enable a proper interaction of CD28 with its signalling partners, allowing a physiological stimulation of CD28 in efficacy studies.

Results hCD28 mice express hCD28 on T cells and the frequency of CD3 T cells is comparable in both WT and hCD28 mice. Stimulation of hCD28 mice-isolated T cells with hCD28 ligands and agonist antibodies resulted in $\mathrm{T}$ cell proliferation and cytokine production, suggesting that hCD28 is functional in mouse cells. MC38 uptake rate and kinetic of growth were comparable in WT and hCD28 mice, suggesting no major defect in the immune response in the hCD28 mice. Importantly, splenocytes and tumor draining lymph nodes cells isolated from tumor-bearing hCD28 mice showed higher production of IL-2 and IFN-gamma upon in vitro re-challenged with MC38 when compared to WT cells. Since the frequency of CD3 cells (Treg, $\mathrm{CD} 4^{+}$and $\mathrm{CD} 8^{+}$) is comparable to WT mice, this could be explained by the expression of the amplifier CD28i isoform, which is absent in WT mice.

Conclusions The hCD28 model described here supports the efficacy assessment of hCD28-targeting biologics, enabling PK/ PD studies as hCD28 expression levels and pattern are physiological. However, after careful consideration of the CD28 biology, we decided to keep the mouse ICD, although it triggers lower pro-inflammatory cytokine production than CD28 human ICD. As such, this model is not suitable for toxicology/safety studies.

\section{REFERENCE}

1. Porciello N, Grazioli P, Campese AF, et al. A non-conserved amino acid variant regulates differential signalling between human and mouse CD28. Nat Commun 2018; 9:1-16.

http://dx.doi.org/10.1136/jitc-2020-SITC2020.0015

\section{ANTIBODY PROFILING OF PROSTATE CANCER PATIENTS REVEALS DIFFERENCES IN ANTIBODY SIGNATURES AMONG DISEASE STAGES AND FOLLOWING TREATMENT}

${ }^{1}$ Hemanth Potluri* ${ }^{1}{ }^{1}$ Tun Lee Ng, ${ }^{1}$ Michael Newton, ${ }^{2}$ Jin Zhang, ${ }^{2}$ Christopher Maher, ${ }^{3}$ Peter Nelson, 'Douglas McNeel. ' UW-Madison, Madison, WI, USA; ${ }^{2}$ Washington University in St. Louis, St. Louis, MO, USA; ${ }^{3}$ Fred Hutchinson Cancer Research Center, Seattle, WA, USA

Background Previous studies of prostate cancer autoantibodies have largely focused on diagnostic applications. So far, there have been no reports attempting to more comprehensively profile the landscape of prostate cancer-associated antibodies. Specifically, it is unknown whether the quantity of antibodies or the types of proteins recognized change with disease progression or treatment.

Methods A peptide microarray spanning the amino acid sequences of the gene products of 1611 prostate cancer-associated genes was synthesized. Serum samples from healthy male volunteers $(n=15)$ and prostate cancer patients $(n=85)$ were used to probe the array. These samples included patients with various clinical stages of disease: newly diagnosed localized prostate cancer, castration-sensitive non-metastatic prostate cancer (nmCSPC), castration-resistant non-metastatic prostate cancer (nmCRPC), and castration-resistant metastatic disease (mCRPC). Serial sera samples from individuals who received treatment with either standard androgen deprivation therapy (ADT) or an anti-tumor vaccine were also used to probe the 